\title{
The mathematical modeling of oscillations in enzymatic systems
}

\author{
Lakhova T.N. ${ }^{1 *}$, Kazantsev F.V. ${ }^{1,2}$, Lashin S.A. ${ }^{1,2}$ \\ ${ }^{1}$ Institute of Cytology and Genetics, SB RAS, Novosibirsk, Russia \\ ${ }^{2}$ Novosibirsk State University, Novosibirsk, Russia \\ *e-mail: tlakhova@bionet.nsc.ru
}

Key words: mathematical model, feedback loop, cycle, oscillatory behavior, microbial enzymatic system

Motivation and Aim: The cyclic processes play essential role in the functioning of biological systems. A lot of such processes are controlled by enzymatic reactions, rates of which may be dramatically influenced by various inhibitors, activators, cofactors, allosteric regulators, etc. It is well-known that the oscillatory behavior in biological systems frequently emerges via influence of the feedback loops. Mathematical modeling is one of the main approaches for the investigation of such complicated systems. Now there are dozens open access databases that store information about biological pathways and Gene Networks that could be the source of data for models of enzymatic reactions. The aim of our research is to develop the technology that can help find potentially oscillating microbial enzymatic subsystems and to explore their behavior.

Methods and Algorithms: The Mammoth database [1] was used as an enzymatic reaction source. Data processing and analysis of structural models were performed with Python. Visual analysis of the structure models was carried out with Cytoscape (https://cytoscape. org/). The mathematical models were reconstructed in terms of ordinary differential equations and computer simulations were processed with Copasi (http://copasi.org). Results: We have analyzed gene network, which consists of 300 E. coli reactions. We have found 10 potential oscillatory cycles. In particular, one in the interest cycles was considered and its oscillatory behavior was proved. This cycle is the part of tryptophan pathway.

\section{References}

1. Kazantsev F. et al. MAMMOTh: a new database for curated MAthematical Models of bioMOlecular system. J. Bioinform. Computational Biol. 2018;16(1):1740010. 